\title{
Análise computacional do dano térmico no olho humano portador de um melanoma de coroide quando submetido à termoterapia transpupilar a laser
}

\author{
José Duarte da Silva*, Paulo Roberto Maciel Lyra, Rita de Cássia Fernandes de Lima
}

\begin{abstract}
Resumo Este trabalho apresenta um modelo bidimensional do olho humano para investigar a evolução da temperatura em estado transitório e o dano térmico associado nas camadas do olho e, principalmente, no melanoma de coroide. A modelagem e simulação computacional foram executadas para um paciente portador de um melanoma de coroide, submetido a um tratamento por irradiação com laser. Para determinar o campo de temperaturas, foi desenvolvido um modelo que utiliza a equação da biotransferência de calor de Pennes (BHTE - Bioheat Transfer Equation) que possui um termo de fonte/sumidouro que responde pelo calor transferido através da perfusão sanguínea. O modelo de Birngruber foi utilizado para determinar a função dano térmico durante a termoterapia transpupilar a laser (TTT). A análise foi efetuada a partir de uma imagem de ultrassom do paciente. Este tipo de exame forneceu as dimensões do olho e do tumor. O software comercial de CFD (Computational Fluid Dynamics) FLUENT, que emprega o Método dos Volumes Finitos (MVF), foi utilizado nas análises do modelo. Foram calculadas as temperaturas em estado estacionário para o olho não irradiado e estes resultados foram utilizados como condição inicial para a simulação de estado transitório para o olho irradiado, durante 60 segundos, com um laser de diodo $(810 \mathrm{~nm}$ e potências de saída de 178 , 222, 400 e $500 \mathrm{~mW}$ ) cujos feixes apresentavam diâmetros de 2,0 e 3,0 mm e intensidades de $56.588 \mathrm{~W} / \mathrm{m}^{2} \mathrm{e}$ $70.736 \mathrm{~W} / \mathrm{m}^{2}$ sobre a córnea. Para validar o modelo, os resultados das temperaturas, em estado estacionário para o olho não exposto à radiação do laser, foram comparados com outros mostrando uma boa concordância entre eles. Os resultados das temperaturas e do dano térmico, estado transitório, para o olho irradiado com o laser estão, qualitativamente, em acordo com a literatura disponível.
\end{abstract}

Palavras-chave Melanoma de coroide, Dano térmico, BHTE, Birngruber, TTT,

Simulação computacional.

\section{Computational analysis of thermal damage in the human eye carrying a choroidal melanoma when exposed to laser transpupillary thermotherapy treatment}

Abstract This paper presents a two-dimensional model of the human eye to investigate the evolution of temperature in transient state and the associated thermal damage in the layers of the eye and, especially in the choroidal melanoma. The computer modeling and simulation were performed for a patient with a choroidal melanoma, undergoing treatment by laser irradiation. To determine the temperature field, we developed a model that uses the Pennes Bioheat Transfer Equation (BHTE) that has a term of source/sink that responds to the heat transferred through the blood perfusion. The Birngruber model was used to determine the function of thermal damage during the laser transpupillary thermotherapy (TTT). The analysis was carried out from an ultrasound image of the patient. This type of examination provided the dimensions of the eye and the tumor. The CFD (Computational Fluid Dynamics) commercial software FLUENT ${ }^{\circledR}$, which uses the Finite Volume Method (FVM) was used in the analysis of the model. The temperatures, steady-state, were calculated for the normal eye, i.e, without laser irradiation and these results were used as initial condition for the simulation of transient state to the eye irradiated for 60 seconds with a diode laser $(810 \mathrm{~nm}$ and output power of 178, 222, 400 and $500 \mathrm{~mW}$ ) whose beam had a diameter of 2.0 to $3.0 \mathrm{~mm}$ and irradiance of 56,588 and $70,736 \mathrm{~W} / \mathrm{m}^{2}$ on the cornea. To validate the model, the results of temperatures, in steady state to the eye not exposed to laser radiation, were compared with others showing a good agreement between them. The results of thermal damage and transient state, for the eye irradiated by the laser, show that the values of damage depth are in agreement with the literature.

Keywords Choroidal melanoma, Thermal damage, BHTE, Birngruber, TTT, Computational simulation.

*e-mail: joduartes@yahoo.com.br

Recebido: 29/12/2011 / Aceito: 10/12/2012 


\section{Introdução}

Os primeiros trabalhos sobre transferência de calor em tecidos biológicos foram efetuados nas primeiras décadas do século XX e focalizaram o transporte de calor entre a pele e o meio ambiente. O modelo da condutividade térmica efetiva considera o transporte de calor como o produto do gradiente de temperatura, entre o corpo e seu entorno, com uma única condutividade térmica que abrange todos os efeitos da vascularização e heterogeneidade das propriedades (Charny, 1992). O principal problema com esta aproximação é que a diferença de temperatura entre o núcleo do corpo e a superfície da pele comanda o escoamento do calor, desprezando a transferência de calor localizada.

Harry H. Pennes (1948) desenvolveu um modelo baseado na difusão de calor e que passou a ser chamado de Equação da Biotransferência de Calor (Bioheat Transfer Equation - BHTE). Segundo ele a transferência de calor nos organismos vivos é caracterizada por dois mecanismos importantes: metabolismo e fluxo sanguíneo. O sangue escoa, de forma não-newtoniana, através dos vasos sanguíneos que apresentam diferentes dimensões. Segundo a teoria de Pennes, a transferência líquida de calor entre o sangue e o tecido é proporcional à diferença entre a temperatura do sangue arterial, que entra no tecido, e a temperatura do sangue venoso que sai do tecido. Ele sugeriu que a transferência de calor devida ao escoamento sanguíneo pode ser modelada por uma taxa de perfusão sanguínea, com o sangue atuando como uma fonte/sumidouro escalar de calor. Apesar da sua simplicidade, uma das dificuldades encontradas no uso da BHTE reside na ausência de informação detalhada e precisa sobre as taxas volumétricas de perfusão sanguínea, especialmente para tecidos neoplásicos.

Wulff (1974) questionou a BHTE argumentando que o calor transferido entre o sangue, que flui nos vasos, e o tecido é proporcional à diferença de temperatura entre estes dois meios, ao invés da diferença de temperatura entre o sangue entrando e o sangue saindo do tecido. Apesar da aparente simplicidade da Equação de Biotransferência de Calor Modificada de Wulff, esta formulação não tem sido tão utilizada quanto a BHTE original, devido às dificuldades em avaliar a velocidade média local do sangue.

Chen e Holmes (1980) desenvolveram um novo modelo para a biotransferência de calor para resolver a crítica que se fazia à equação de Pennes. Este modelo foi uma tentativa de incorporar os efeitos do escoamento sanguíneo na equação da transferência de calor de modo a captar a direção do escoamento sanguíneo e incluir características da transferência de calor por convecção na transferência de calor entre o sangue e o tecido sólido. $\mathrm{O}$ modelo considera que o volume de controle é composto por um subvolume sólido (tecido) e por um subvolume líquido (sangue). Apesar de ser um modelo mais realístico do que o modelo de Pennes, ele requer parâmetros e termos que, geralmente, não estão disponíveis. Por exemplo, a falta de conhecimento sobre o vetor velocidade do sangue. Deve ser registrado, também, que foram feitas considerações sobre o escoamento e fisiologia que não são totalmente válidas. Eles ignoraram um importante aspecto da transferência de calor em tecidos biológicos, responsável pela regulação térmica: o escoamento em sentidos contrários do par artéria-veia e a consequente natureza contracorrente da troca de calor nos tecidos biológicos.

Weinbaum et al. (1984) trocaram a condutividade térmica na equação da biotransferência de calor de Pennes por uma "condutividade efetiva" com representação tensorial. O modelo matemático desenvolvido por Weinbaum, Jiji e Lemons é complexo e pode ser encontrado em detalhes em seu próprio trabalho ou em Charny (1992). Esta formulação está baseada na hipótese de que pequenas artérias e veias são paralelas com o sangue escoando em sentidos contrários. Vale salientar que este modelo apresenta uma forte dependência da configuração dos vasos sanguíneos e exige informações fisiológicas detalhadas e precisas para uma análise apropriada. Contudo, esta informação fisiológica não existe, fato este que restringe o uso de mais essa formulação.

Dessa maneira, pode-se afirmar que, as várias tentativas de aperfeiçoar o modelo da biotransferência de calor de Pennes resultaram em modelos com maior rigor na análise matemática, alta complexidade e reduzida generalidade. Por estas razões e devido à sua simplicidade, a equação de Pennes continua sendo a mais utilizada para descrever a transferência de calor e a distribuição da temperatura em tecidos biológicos vivos.

Quanto ao melanoma de coroide, é o câncer ocular mais comum, com incidência aproximada de 4,9 casos por milhão em homens e 3,7 casos por milhão em mulheres por ano nos EUA, segundo Singh (Cunha et al., 2010). Acomete preferencialmente indivíduos acima dos 50 anos de idade e da raça branca, sendo raro nas raças negra e asiática. Em alguns pacientes ele é assintomático, enquanto em outros pode causar perda de visão, defeitos no campo visual, fotopsia ou dor (Arcieri et al., 2002).

A escolha do tratamento do melanoma de coroide ainda é controversa em muitos aspectos e o melhor procedimento terapêutico ainda não está estabelecido. 
Algumas das principais opções terapêuticas são: enucleação, exenteração, ressecção cirúrgica localizada, braquiterapia, irradiação por feixe externo de partículas carregadas, fotocoagulação e termoterapia transpupilar a laser (Valenzuela, 2009). A remoção de tumores através de cirurgia é um processo doloroso, traumatizante e algumas vezes inaplicável, por isso, o tratamento hipertérmico denominado termoterapia transpupilar a laser tem despertado grande interesse e apresentado resultados satisfatórios para determinados tipos de tumores.

A termoterapia transpupilar (TTT) é um método que utiliza radiação infravermelha como fonte de calor, provocando o aquecimento do tecido tumoral (Shields et al., 1998). A absorção da radiação do laser de diodo pelo meio ocular é muito baixa para induzir efeitos nesta região (Van den Berg e Spekreijse, 1997). Desta forma, o laser é quase que totalmente absorvido pela melanina presente em altas concentrações na maioria dos melanomas (Newsom et al., 2001). Esta absorção causa um aumento na temperatura tumoral que pode atingir valores entre $45^{\circ} \mathrm{C}$ e $60^{\circ} \mathrm{C}$ resultando em necrose de tecido (Stoffelns, 2002).

O tempo de aplicação é função da potência do laser, sendo determinado experimentalmente pela própria reação tecidual, ou seja, quando começa a ocorrer uma discreta mudança na coloração do melanoma, sendo o laser desligado neste momento (Roizenblatt e Rosa, 2002). Tal procedimento é insatisfatório, pois pode induzir a erros por parte dos médicos que executam o tratamento. Portanto, a simulação numérica desses procedimentos pode ser fundamental, fornecendo parâmetros confiáveis como tempo de exposição e potência utilizada pelo laser, para conduzir o tratamento com o mínimo risco para o paciente.

Dano no tecido é definido como a desnaturação das proteínas ou a perda das funções biológicas das moléculas encontradas nas células ou em fluidos extracelulares (Diller, 1992). Esse dano térmico pode ocorrer quando a temperatura do tecido excede o intervalo dentro do qual podem existir os processos normais da vida. Tanto temperaturas elevadas quanto baixas podem causar alterações irreversíveis nas moléculas, resultando em dano térmico. Exemplos comuns são queimaduras e congelamento de pele. Os modelos matemáticos, de maior sucesso, consideram que o dano térmico é uma reação química que depende da temperatura e do intervalo de tempo ao qual o tecido vivo ficou submetido a ela. Os modelos descrevem o efeito do calor na taxa de reação química, e consideram a variação na concentração de moléculas não danificadas, utilizando a lei de Arrhenius. Apesar de o significado da função dano térmico não estar muito bem definido, foi considerado que quando a função dano assume o valor um $\left(\Omega_{\mathrm{D}}=1\right)$, que corresponde a, aproximadamente, $63 \%$ das proteínas desnaturadas, ocorreu uma necrose completa, ou dano irreversível do tecido biológico (Henriques e Moritz, 1947).

Portanto, neste trabalho é desenvolvido um modelo físico-matemático e computacional para determinar a temperatura, em regime estacionário e em regime transitório, e seus efeitos no olho humano quando submetido à radiação de um laser. Este modelo será utilizado, também, para avaliar o dano térmico causado ao tecido ocular, principalmente no melanoma, e posteriormente, fornecer uma estimativa do intervalo de tempo necessário para a radiação, com uma determinada irradiância, produzir danos térmicos irreversíveis ao tecido tumoral, causando os menores prejuízos possíveis aos tecidos sadios nos procedimentos de termoterapia transpupilar (TTT). Neste estudo será considerado um modelo bidimensional do olho e a análise será executada a partir de uma imagem de ultrassom realizado no olho de um paciente portador de um melanoma de coroide, onde existem informações sobre as dimensões do olho e do tumor.

\section{Materiais e Métodos}

\section{Modelo físico e matemático}

Devido à sua simplicidade, à ausência de parâmetros adicionais necessários em modelos mais complexos e aos bons resultados obtidos pela Equação de Pennes, esta equação foi escolhida para determinar a temperatura nas várias camadas do olho portador de um melanoma de coroide quando irradiado com laser contínuo de diodo (comprimento de onda igual a $810 \mathrm{~nm}$ e potências de saída de $178,222,400$ e $500 \mathrm{~mW}$ ). A equação pode ser escrita como:

$\rho_{t} c_{t} \frac{\partial T_{t}}{\partial t}=\nabla\left(k_{t} \nabla T_{t}\right)+Q_{P}+Q_{m}+Q_{L}$

onde,

$Q_{P}=\omega \rho_{b} c_{b}\left(T_{a}-T_{t}\right)$

A Equação 1 representa uma equação de difusão de calor com particularidades no termo fonte, que é desdobrado em três partes: $Q_{P}$ que representa uma fonte/sumidouro de calor devido à perfusão sanguínea; $Q_{m}$ que representa a geração de calor metabólico, desprezada por ser muito menor que a geração de calor devida ao laser; $Q_{L}$ que representa a taxa volumétrica de energia depositada no tecido pela radiação do laser. 
Nesse trabalho não foi considerado o espalhamento da radiação no meio, devido à falta de informações confiáveis sobre esse parâmetro e devido às incertezas sobre as relações entre os coeficientes de absorção e de espalhamento para os vários tecidos oculares. Então, por conta da complexidade inerente a uma análise da distribuição da energia espalhada no meio, foi assumido, como uma primeira aproximação, que a energia depositada é devida apenas à absorção da energia do laser e ocorre de acordo com a lei de Lambert-Beer com $Q_{\mathrm{L}}=\beta I_{0} \exp (-\beta x)$ (Shitzer, 1985). Desta forma a intensidade do laser decai exponencialmente ao longo da direção de propagação de um feixe cilíndrico de radiação que atravessa um meio homogêneo. O primeiro termo do lado direito representa a taxa volumétrica do calor transportado por condução e o termo do lado esquerdo representa a variação da taxa volumétrica de calor no volume de controle.

\section{Nomenclatura}

- $c$ calor específico $\left(\mathrm{J} \cdot \mathrm{kg}^{-1} \mathrm{~K}^{-1}\right)$

- $C$ constante pré-exponencial $\left(\mathrm{s}^{-1}\right)$

- $I_{0}$ intensidade do laser na córnea $\left(\mathrm{W} \cdot \mathrm{m}^{-2}\right)$

- $k$ condutividade térmica $\left(\mathrm{W} \cdot \mathrm{m}^{-1} \mathrm{~K}^{-1}\right)$

- $Q$ taxa volumétrica de calor $\left(\mathrm{W} \cdot \mathrm{m}^{-3}\right)$

- $R$ constante universal dos gases $\left(\mathrm{J} \cdot \mathrm{mol}^{-1} \mathrm{~K}^{-1}\right)$

- $T$ temperatura $(\mathrm{K})$

- $\bar{T}$ temperatura prescrita $(\mathrm{K})$

- $\bar{T}^{i}$ temperatura inicial $(\mathrm{K})$

- I intervalo de tempo de integração (s)

- $t$ tempo (s)

- $t^{\mathrm{i}}$ instante de tempo inicial (s)

- $t^{\mathrm{f}}$ instante de tempo final (s)

- $x$ distância percorrida pela radiação $(\mathrm{m})$

- $y$ ordenada cartesiana (m)

Símbolos gregos

- $\Gamma_{\mathrm{C}}$ superfície anterior da córnea

- $\Gamma_{\mathrm{D}}$ superfície posterior da esclera

- $\beta$ coeficiente de absorção $\left(\mathrm{m}^{-1}\right)$

- $\Delta E_{\text {at }}$ energia de ativação $\left(\mathrm{J} \cdot \mathrm{mol}^{-1}\right)$

- $\rho$ densidade $\left(\mathrm{kg} \cdot \mathrm{m}^{-3}\right)$

- $\tau$ tempo de desnaturação (s)

- $\omega$ taxa de perfusão $\left(\mathrm{s}^{-1}\right)$

- $\Omega_{\mathrm{D}}$ dano térmico (adimensional)

- $\Omega$ volume de controle

- $\eta$ vetor normal unitário (m)

Subscritos

- $a$ arterial

- $b$ sangue

- $c$ córnea
- $L$ laser

- $m$ metabólico

- $\quad P$ perfusão

- $t$ tecido biológico

A Figura 1 mostra um esquema para o olho de um paciente com melanoma de coroide como uma estrutura composta por diferentes tecidos oculares. Para simplificar a modelagem, foram consideradas as seguintes hipóteses e condições de contorno e inicial:

a) O olho é uma estrutura sólida composta por camadas diferentes em contato;

b) O laser incidente apresenta o padrão spot, i.e., com intensidade que não varia radialmente dentro do feixe;

c) A radiação laser penetra no tecido sem sofrer espalhamento;

d) Os diferentes tecidos são homogêneos e isotrópicos;

e) A superfície da córnea troca calor por convecção e por radiação com o ambiente;

f) Não foi considerada a geração de calor metabólico $\left(\mathrm{Q}_{\mathrm{m}}<<\mathrm{Q}_{\mathrm{L}}\right)$;

g) A retina e o tumor foram considerados como uma única região, aqui denominada de tumor, porque a retina possui uma espessura muito pequena e também porque a maior parcela da radiação infravermelha é absorvida no tecido do tumor rico em melanina;

h) As propriedades físicas da íris e do corpo ciliar são idênticas às do humor aquoso (Amara, 1995). Portanto, estas regiões foram tratadas como uma única região aqui denominada humor aquoso;

i) O humor aquoso foi considerado estagnado (Emery et al., 1975; Kramar et al., 1978, Scott, 1988);

j) A transferência de calor dentro do olho ocorre por condução;

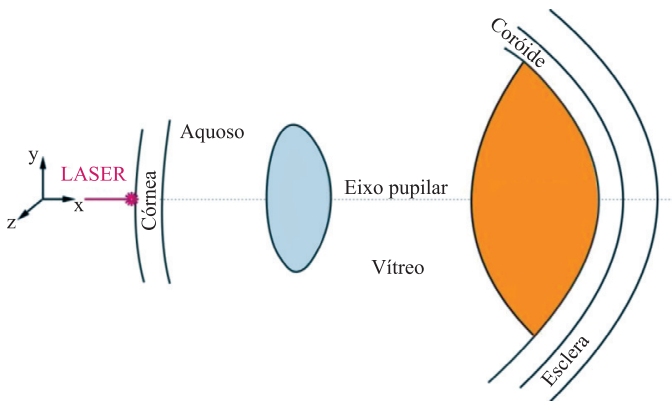

Figura 1. Representação simplificada das camadas do olho. Figure 1. Simplified representation of the eye layers. 
k) A perfusão sanguínea foi considerada apenas no tumor e na coroide;

1) $\mathrm{O}$ feixe de laser foi considerado cilíndrico e atinge a córnea numa direção paralela ao eixo pupilar, passando pelo centro óptico. Não foi considerada a ação focalizadora do cristalino;

m) Uma condição de contorno de temperatura constante foi imposta para a superfície posterior da esclera;

n) Uma condição de contorno convectiva foi imposta sobre a superfície anterior do olho com coeficiente de transferência de calor estimado por Lagendijk (Scott, 1988) considerando a evaporação do filme lacrimal e a troca de calor por radiação e convecção para o ambiente $\operatorname{com} h=(20 \pm 2) \mathrm{W} / \mathrm{m}^{2}{ }^{\circ} \mathrm{C}$;

o) As temperaturas iniciais nas diferentes regiões do olho foram obtidas por meio de uma simulação preliminar em regime estacionário para o olho não exposto à radiação; e

p) Os eixos pupilar, visual e óptico foram considerados como coincidentes devido à simetria do problema.

Para determinar o valor do dano térmico causado ao tecido nas várias camadas do olho, será utilizado o modelo que Birngruber (Rol et al., 2000) desenvolveu para determinar a função dano na retina e na coroide durante tratamentos com termoterapia transpupilar a laser. O modelo descreve o efeito do calor na taxa de reação química, e considera a variação na concentração de moléculas não danificadas, utilizando a lei de Arrhenius. A equação pode ser escrita como:

$\Omega_{\mathrm{D}}=C \int_{0}^{\tau} T(t) \exp \left(-\frac{\Delta E_{a t}}{R T(t)}\right) d t$

A Equação 3 difere dos modelos tradicionais pelo valor da constante pré-exponencial, $C$, e pelo fato de a temperatura, $T(t)$, estar presente no integrando da mesma.

A Figura 2 mostra um esquema para o olho humano portador de um melanoma de coroide, construído a partir de uma imagem de ultrassom, dividido em sete regiões. O diâmetro ao longo do eixo pupilar é de aproximadamente $24 \mathrm{~mm}$ (Adler, 1970; L'Huillier e Apiou-Sbirlea, 2000 apud Narasimhan et al., 2009). A metade posterior do globo ocular humano é quase esférica (Forrester et al., 2002). Cada região é considerada homogênea e o olho pode ser considerado simétrico com relação ao eixo pupilar, desde que se despreze a existência do nervo óptico.

A córnea é considerada com uma espessura constante e igual a $0,5 \mathrm{~mm}$ (Smerson, 2000). O cristalino tem um diâmetro de $8,4 \mathrm{~mm}$ e uma espessura

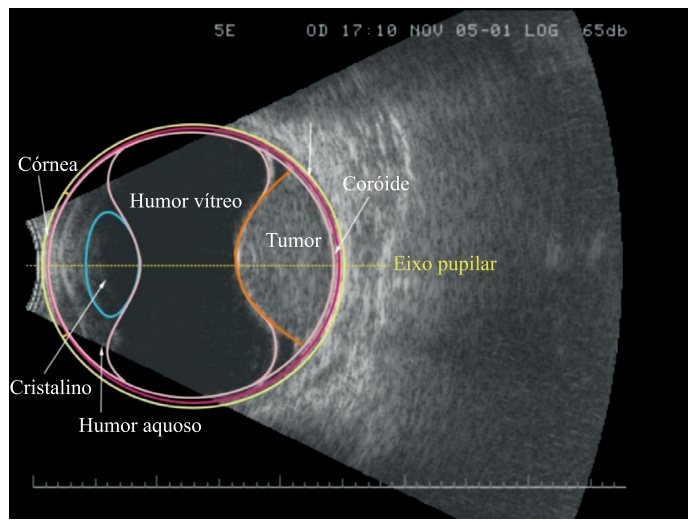

Figura 2. Um esquema para o modelo do olho com um melanoma real construído a partir de uma imagem de ultrassom do olho de um paciente.

Figure 2. A scheme for the eye model with a real melanoma constructed from an ultrasound image of the eye of a patient.

de 4,3 mm. A espessura da esclera é considerada constante e aproximadamente igual a $0,6 \mathrm{~mm}$ (Narasimhan et al., 2009). O diâmetro do tumor é $14,2 \mathrm{~mm}$ e a sua espessura é de $7,9 \mathrm{~mm}$. A coroide foi considerada com uma espessura aproximadamente constante de $0,5 \mathrm{~mm}$.

A condutividade térmica, a densidade, o calor específico, a taxa de perfusão sanguínea e o coeficiente de absorção são considerados constantes para cada região do olho. As propriedades termofísicas da esclera foram consideradas iguais às da córnea; as propriedades termofísicas e a taxa de perfusão sanguínea da coroide foram consideradas iguais às da retina. Valores para estas propriedades podem ser encontradas na literatura e são apresentados na Tabela 1 .

A equação governante do problema é a equação da biotransferência de calor de Pennes (Equação 1), descrita anteriormente. Para a análise bidimensional, serão utilizadas coordenadas cartesianas e a Equação 1 pode ser escrita como:

$\rho_{t} c_{t} \frac{\partial T_{t}}{\partial t}=\frac{\partial}{\partial x}\left(k_{t} \frac{\partial T_{t}}{\partial x}\right)+\frac{\partial}{\partial y}\left(k_{t} \frac{\partial T_{t}}{\partial y}\right)+Q \quad$ em $\quad \Omega$ X I

Onde $Q$ representa os termos de fonte/sumidouro de calor. O domínio espacial do problema é representado por $\Omega$ e $\mathbf{I}=\left[t^{\mathrm{i}}, t^{\mathrm{f}}\right]$ representa o intervalo de tempo da integração.

Para resolver a Equação 4, que é uma equação diferencial parcial linear de segunda ordem, parabólica e não homogênea, no domínio do olho foram impostas as seguintes condições inicial e de contorno:

Condição de contorno de Dirichlet:

$T=\bar{T} \quad$ em $\quad \Gamma_{D} \mathrm{XI}$ 
Tabela 1. Coeficientes de absorção, taxas de perfusão e propriedades termofísicas.

Table 1. Absorption coefficient, perfusion rate and thermophysical properties.

\begin{tabular}{cccccc}
\hline Camada & $\rho\left(\mathbf{k g} / \mathbf{m}^{3}\right)$ & $\mathbf{c}\left(\mathbf{J} / \mathbf{k g}^{\circ} \mathbf{C}\right)$ & $\mathbf{k}\left(\mathbf{W} / \mathbf{m}^{\circ} \mathbf{C}\right)$ & $\beta\left(\mathbf{m}^{-1}\right)$ & $\omega\left(\mathbf{s}^{-1}\right)$ \\
\hline Aquoso & 1000 & 3997 & 0,58 & 16,82 & - \\
Córnea & 1050 & 4178 & 0,58 & 120,52 & - \\
Coroide & 1000 & 4190 & 0,628 & 1377,88 & 0,012 \\
Esclera & 1050 & 4178 & 0,58 & 120,52 & - \\
Lente & 1050 & 3000 & 0,40 & 20,26 & - \\
Tumor & 1040 & 3900 & 0,70 & 1377,88 & 0,00399 \\
Vítreo & 1000 & 4178 & 0,603 & 7,69 & - \\
\hline
\end{tabular}

Fonte: Amara (1995), Cheong et al. (1990), Rivolta et al. (1999) e Welch (1985). ${ }^{*}$ Os valores para o coeficiente de absorção nas diversas camadas do olho para a radiação infravermelha do laser de diodo $(810 \mathrm{~nm})$ foram obtidos por meio de interpolação linear utilizando-se os dados encontrados na literatura para esses coeficientes. Para córnea, humor aquoso, cristalino e humor vítreo foram encontrados valores em Amara (1995) para os comprimentos de onda 694,3 nm e $1060 \mathrm{~nm}$; Para a esclera foram considerados os mesmos valores utilizados para a córnea; Para o melanoma, a interpolação foi feita com os valores do coeficiente de atenuação para melanoma em ratos (Cheong et al., 1990), comprimentos de onda $630 \mathrm{~nm}$ e $1064 \mathrm{~nm}$; Para a coroide, foi assumido um valor igual ao do tumor.

Condição de contorno de Cauchy, ou de Robin, ou mista:

$k \frac{\partial T_{t}}{\partial \eta}=h_{c}\left(T_{t}-T_{a}\right) \quad$ em $\quad \Gamma_{\mathbf{C}} \mathrm{XI}$

Condição inicial: é conhecida a distribuição de temperatura $T$ para um estágio de tempo i-nicial $t^{i}$, expressa por:

$T=\bar{T}^{i}$ em $\Omega \quad$ e $\quad t=t^{i}$

As Equações 5 a 8 descrevem completamente o modelo matemático proposto, que governa o transporte de calor no meio.

\section{Método numérico e estudo de convergência de malha}

No domínio do problema aqui analisado foram usadas malhas não-estruturadas devido à facilidade da adequação dessas malhas para geometrias complexas, permitindo ainda que refinamentos locais possam ser feitos em regiões específicas. Malhas não-estruturadas são adequadas para a discretização de geometrias quaisquer, já a malhas estruturadas requerem que a geometria seja descrita por quatro faces, permitindo o mapeamento da mesma em um retângulo. Nas malhas estruturadas, diferentemente das não-estruturadas, cada volume interno tem sempre o mesmo número de vizinhos, a numeração dos mesmos tem uma sequência natural e a matriz resultante possui diagonais fixas que são mais fáceis de implementar. Vários exemplos de comparação entre estes dois tipos de malha podem ser encontrados em Maliska (1995).

Modelos da malha para o olho foram criados usando o programa comercial Gambit ${ }^{\circledR}$. As coordenadas dos pontos da imagem, utilizadas pelo Gambit ${ }^{\mathbb{R}}$ na geração da malha, foram adquiridas por meio do programa computacional APID - Aquisição de Pontos em Imagens Digitais - (Santos, 2007). São usados elementos triangulares para a malha no domínio do olho com refinamento na região do tumor, onde são esperadas as maiores e as mais rápidas mudanças de temperatura. O programa comercial de CFD (Computational Fluid Dynamics) Fluent ${ }^{\circledR}$ (http://www.ansys.com), que emprega o Método dos Volumes Finitos (MVF), foi utilizado para calcular as temperaturas. Os cálculos foram efetuados utilizando-se uma formulação implícita com um esquema upwind de segunda ordem para as equações de energia. O dano térmico causado no olho e no tumor foi calculado por meio de uma rotina escrita em MATLAB ${ }^{\circledR}$ utilizando as temperaturas calculadas a cada passo no tempo para alguns pontos de interesse, denominados a seguir: $x_{0}=$ córnea, $x_{\mathrm{a}}=$ humor aquoso, $x_{1}=$ cristalino, $x_{\mathrm{v}}=$ humor vítreo, $x_{\mathrm{t}}=$ tumor, $x_{\mathrm{c}}=$ coroide $\mathrm{e} x_{\mathrm{e}}=$ esclera. Estes pontos, todos, situam-se na intersecção do eixo pupilar com a superfície anterior da referida camada ocular.

Foi realizado estudo de convergência da malha para o olho irradiado com laser. Em regime transitório, foram impostos: intensidade do laser igual a $70.736 \mathrm{~W} / \mathrm{m}^{2}$; diâmetro do feixe de laser igual a $3,0 \mathrm{~mm}$; temperatura de $37^{\circ} \mathrm{C}$ para o corpo humano e uma temperatura ambiente de $25^{\circ} \mathrm{C}$. O resultado deste estudo é mostrado na Tabela 2. Tomando como referência o resultado obtido com a malha contendo 104.186 elementos, considerada como a que fornece resultados mais próximos da solução real, entre todas as malhas mostradas, foi escolhida uma com 41.680 elementos para as demais simulações, pois os resultados obtidos com essa malha apresentaram diferenças de $0,4817 \%$ na temperatura da córnea $\left(T_{0}\right)$, de $0,0477 \%$ na temperatura do tumor $\left(T_{t}\right)$ e $0,0026 \%$ na temperatura da esclera $\left(T_{\mathrm{e}}\right)$ em relação aos valores obtidos com a malha de referência. Os pontos, onde 
estas temperaturas foram tomadas, situam-se na interseção do eixo pupilar com a superfície anterior da referida camada ocular.

\section{Resultados}

Inicialmente foi analisada a transferência de calor no olho não exposto à radiação do laser. Foram determinadas as temperaturas em regime estacionário para todos os pontos do domínio de acordo com as condições mostradas na Tabela 3. Estes resultados foram utilizados como valores iniciais para os cálculos do estado transitório para o olho exposto à radiação do laser.

A Figura 3 mostra o campo de temperaturas obtido para o olho humano portador de um melanoma de coroide antes da aplicação do laser. Pode ser observado que o campo de temperaturas apresenta uma diminuição na sua intensidade à medida que se aproxima da córnea. Este comportamento é devido às condições de contorno impostas que incluem troca de calor por convecção com o ambiente. A córnea cobre uma área frontal do olho com diâmetro de $10 \mathrm{~mm}$ e perde calor para o ambiente. A esclera cobre toda a superfície do olho exceto aquela correspondente à córnea, e possui temperatura constante (condição de contorno).

Para validar o nosso modelo, os resultados da distribuição de temperatura no olho não irradiado foram comparados com os obtidos por Amara (1995) e por Ng e Ooi (2006) que efetuaram simulações em condições semelhantes. Estes resultados são apresentados na Figura 4 e mostram uma boa concordância entre eles. As temperaturas na superfície anterior da córnea, calculadas pelos vários modelos, são as que apresentaram as maiores diferenças. Encontrou-se uma diferença de $0,53{ }^{\circ} \mathrm{C}$ entre o modelo atual e o modelo de Amara, contudo foi encontrada uma diferença de $0,2{ }^{\circ} \mathrm{C}$, quando a comparação foi feita com o modelo mais recente de $\mathrm{Ng}$ e Ooi (2006).

O campo de temperaturas para o olho irradiado com laser de intensidade $70.736 \mathrm{~W} / \mathrm{m}^{2}$ e diâmetro do feixe igual a 2,0 mm é mostrado na Figura 5. Diferentemente da Figura 3, aqui a distribuição de temperaturas segue outro padrão. Esta diferença é
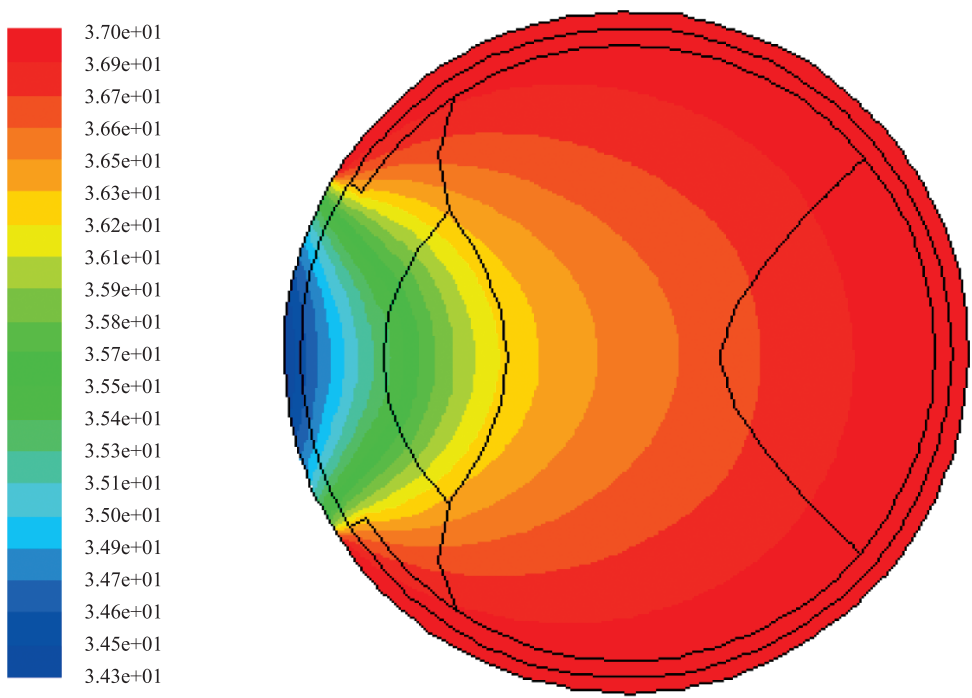

Figura 3. Campo de temperaturas em estado estacionário para o olho humano não exposto à radiação do laser.

Figure 3. Temperature field in steady-state for the human eye without laser radiation.

Tabela 2. Testes para convergência da malha.

Table 2. Grid convergence tests.

\begin{tabular}{|c|c|c|c|c|c|}
\hline Teste & $\begin{array}{l}\text { Número de } \\
\text { elementos }\end{array}$ & $\mathrm{T}_{1}\left({ }^{\circ} \mathrm{C}\right)$ córnea & $\mathrm{T}_{2}\left({ }^{\circ} \mathrm{C}\right)$ tumor & $\mathrm{T}_{3}\left({ }^{\circ} \mathrm{C}\right)$ esclera & RMS(\%) \\
\hline 1 & 3.458 & 53,961 & 84,174 & 38,014 & 0,5060 \\
\hline 2 & 11.702 & 53,982 & 83,915 & 37,991 & 0,3872 \\
\hline 3 & 41.680 & 54,128 & 83,805 & 37,993 & 0,2479 \\
\hline 4 & 72.124 & 54,348 & 83,621 & 37,981 & 0,2135 \\
\hline 5 & 104.186 & 54,390 & 83,845 & 37,994 & - \\
\hline
\end{tabular}


devida ao calor depositado no meio pela radiação do laser. Pelo fato de o coeficiente de absorção do tumor ser maior do que para as outras camadas do olho, a radiação infravermelha praticamente não é

Tabela 3. Valores dos parâmetros de controle usados nas simulações. Table 3. Values of the control parameters used in the simulations.

\begin{tabular}{ccc}
\hline Parâmetros de controle & Valor & Referência \\
\hline Temperatura do sangue & $37{ }^{\circ} \mathrm{C}$ & $\begin{array}{c}\mathrm{Ng} \text { e Ooi } \\
(2006)\end{array}$ \\
Temperatura ambiente & $25{ }^{\circ} \mathrm{C}$ & $\begin{array}{c}\mathrm{Ng} \mathrm{e} \mathrm{Ooi} \\
(2006)\end{array}$ \\
$\begin{array}{c}\text { Temperatura da superfície } \\
\text { posterior da esclera }\end{array}$ & $37^{\circ} \mathrm{C}$ & - \\
$\begin{array}{c}\text { Coeficiente de transferência } \\
\text { de calor córnea/ambiente }\end{array}$ & $22 \mathrm{~W} / \mathrm{m}^{2}{ }^{\circ} \mathrm{C}$ & $\begin{array}{c}\text { Lagendijk } \\
(1982)\end{array}$ \\
\hline
\end{tabular}

absorvida nas outras camadas, a temperatura atinge os mais altos valores nesta região, como esperado.

A variação da temperatura ao longo do eixo pupilar após 1 minuto de exposição do olho à radiação de intensidade $70.736 \mathrm{~W} / \mathrm{m}^{2}$ e diâmetro do feixe do laser igual a $2,0 \mathrm{~mm}$ é mostrada na Figura 6 , onde se observa que as temperaturas mais altas estão localizadas dentro do tumor, próximo à superfície. Este fato pode ser explicado pelo alto valor do coeficiente de absorção pelo tecido melanótico do tumor. As pequenas variações da temperatura na base do tumor (na posição $\approx 2,2 \mathrm{~cm}$ ) estão em acordo com a literatura (Journée-de Korver et al., 1997), onde se afirma que a redução no tamanho do tumor não é efetiva nesta área. Portanto, não ocorrem efeitos hipertérmicos satisfatórios nessa região.

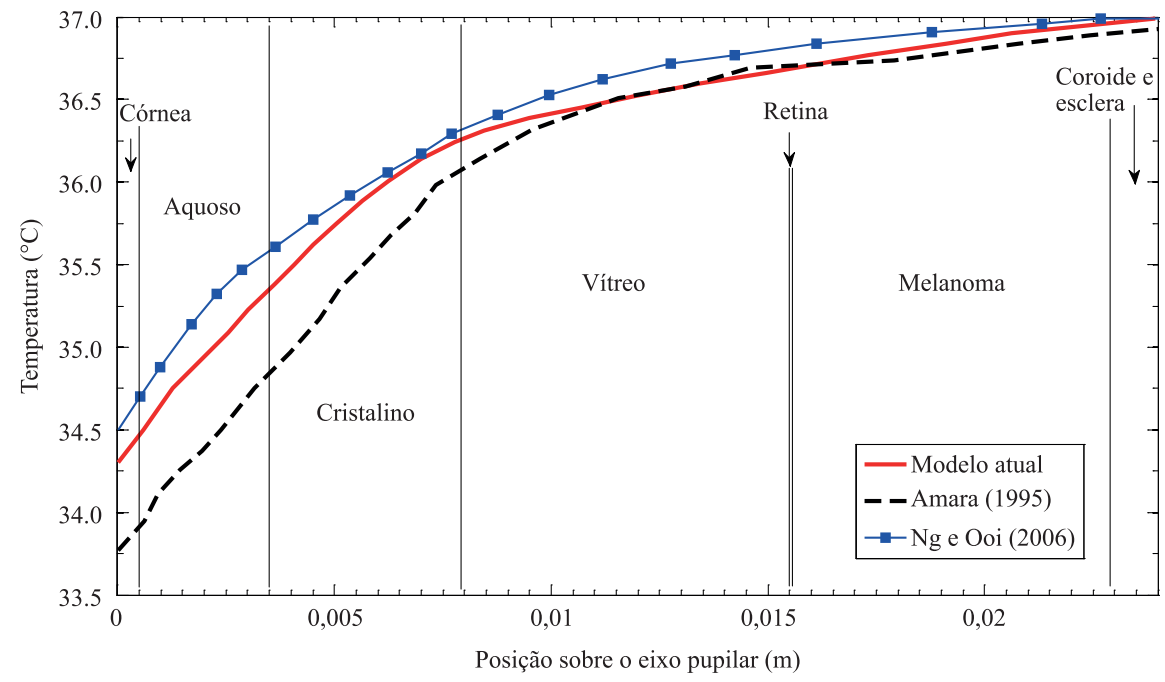

Figura 4. Distribuição espacial da temperatura sobre o eixo pupilar em estado estacionário para o olho não exposto à radiação do laser. Figure 4. Spatial distribution of the temperature on the pupillary axis in the steady-state case without laser irradiation.
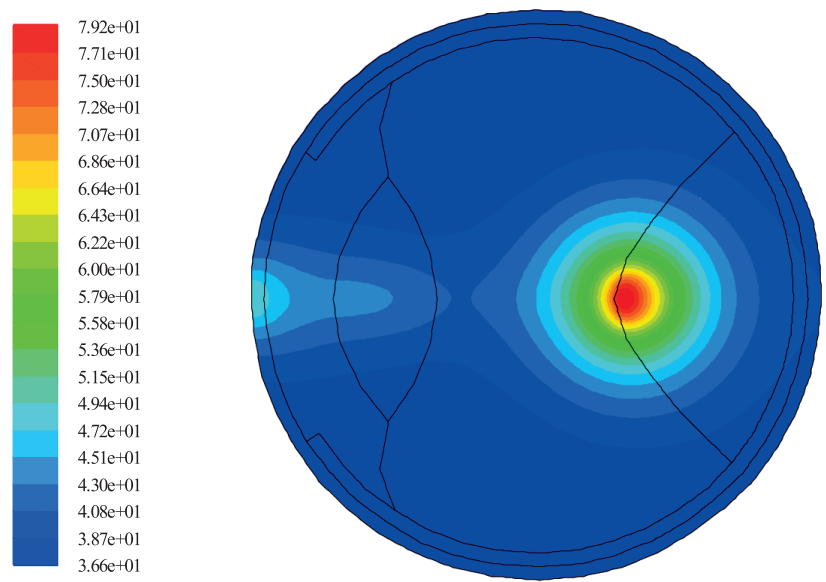

Figura 5. Campo de temperaturas para o olho exposto durante 60 segundos à radiação do laser com intensidade igual a $70.736 \mathrm{~W} / \mathrm{m}^{2}$. Figure 5. Temperature field for the eye exposed for 60 seconds to laser radiation with an intensity of 70,736 W/m2. 
A Figura 7 mostra o dano térmico causado pela radiação de um laser com intensidade de $56.588 \mathrm{~W} / \mathrm{m}^{2}$ em função do tempo para dois diferentes valores do diâmetro do feixe de laser. Uma análise desse gráfico revela que para o diâmetro de 3,0 mm o valor máximo do dano é aproximadamente cinco vezes maior do que para a irradiação com laser com 2,0 mm de diâmetro. Este aumento no valor do dano é resultante da maior quantidade de energia entregue ao tecido; para um diâmetro do feixe de laser igual a $3,0 \mathrm{~mm}$ o tecido recebeu 2,25 vezes mais energia do que com diâmetro igual a 2,0 mm. É evidente, também, que o valor do dano continuou aumentando durante uns poucos segundos após o laser ter sido desligado (em $\mathrm{t}=60$ segundos) e em seguida atingiu um valor constante.

A Figura 8 representa o dano total, no instante de tempo $t=120$ segundos, em função da distância sobre o eixo pupilar para a radiação do laser com intensidades

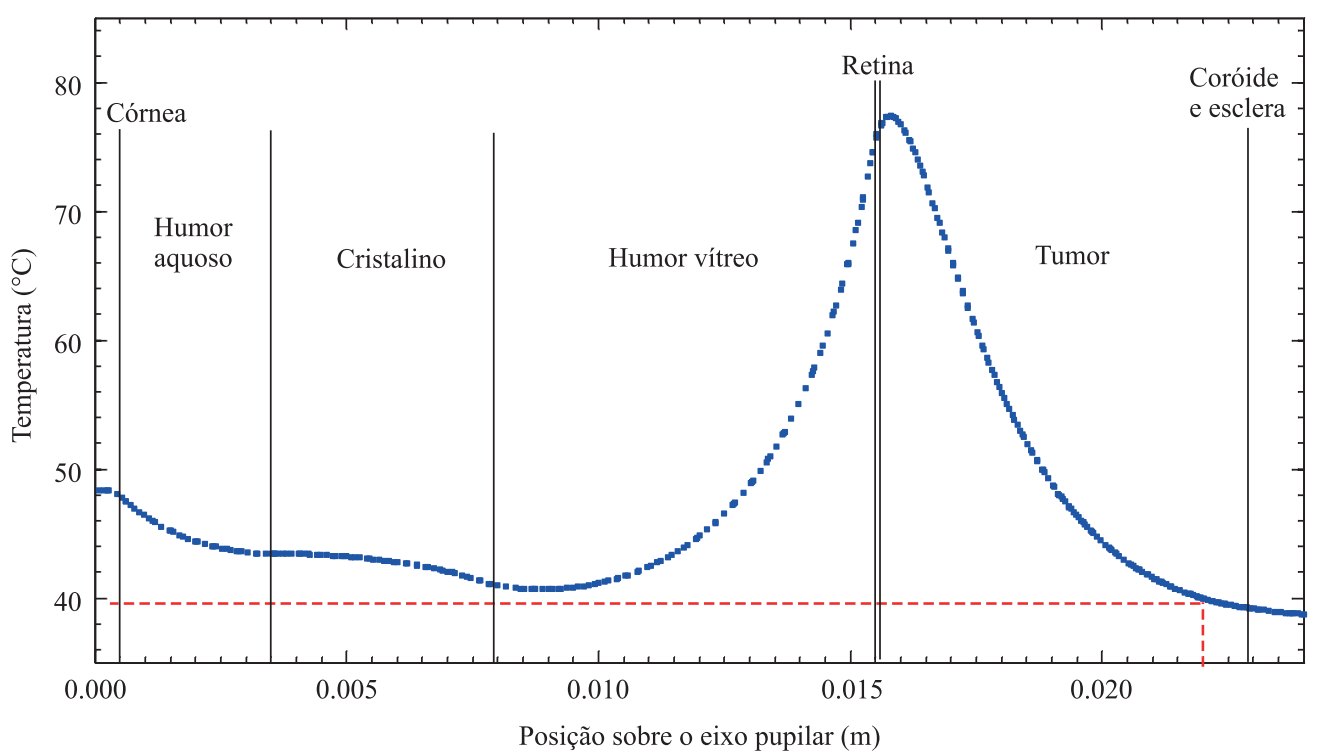

Figura 6. Distribuição espacial da temperatura sobre o eixo pupilar, em estado transitório, após 60 segundos de irradiação com laser de intensidade $70.736 \mathrm{~W} / \mathrm{m}^{2}$.

Figure 6. Spatial distribution of the temperature on the papillary axis, in the transient state, after 60 seconds of laser irradiation with an intensity of $70,736 \mathrm{~W} / \mathrm{m}^{2}$.

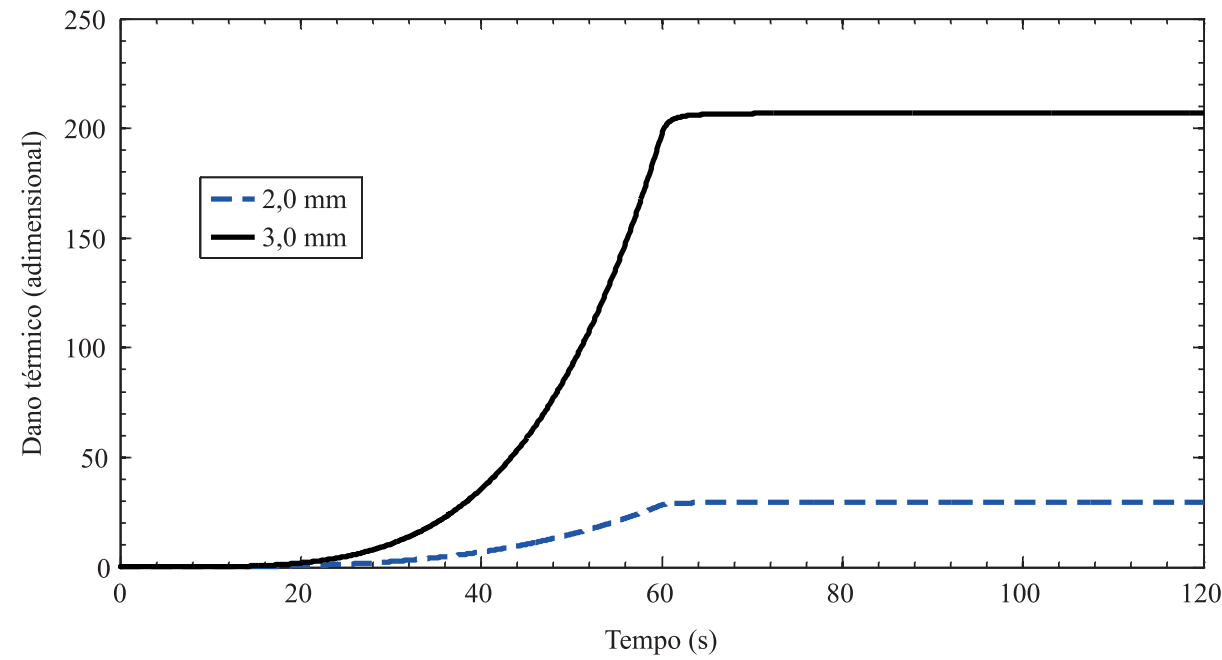

Figura 7. Evolução temporal do dano térmico na superfície do tumor para o olho irradiado durante 60 segundos com laser de intensidade igual a $56.588 \mathrm{~W} / \mathrm{m}^{2}$.

Figure 7. Temporal evolution of thermal damage on the surface of the tumor to the eye irradiated for 60 seconds with laser intensity equal to $56,588 \mathrm{~W} / \mathrm{m}^{2}$. 


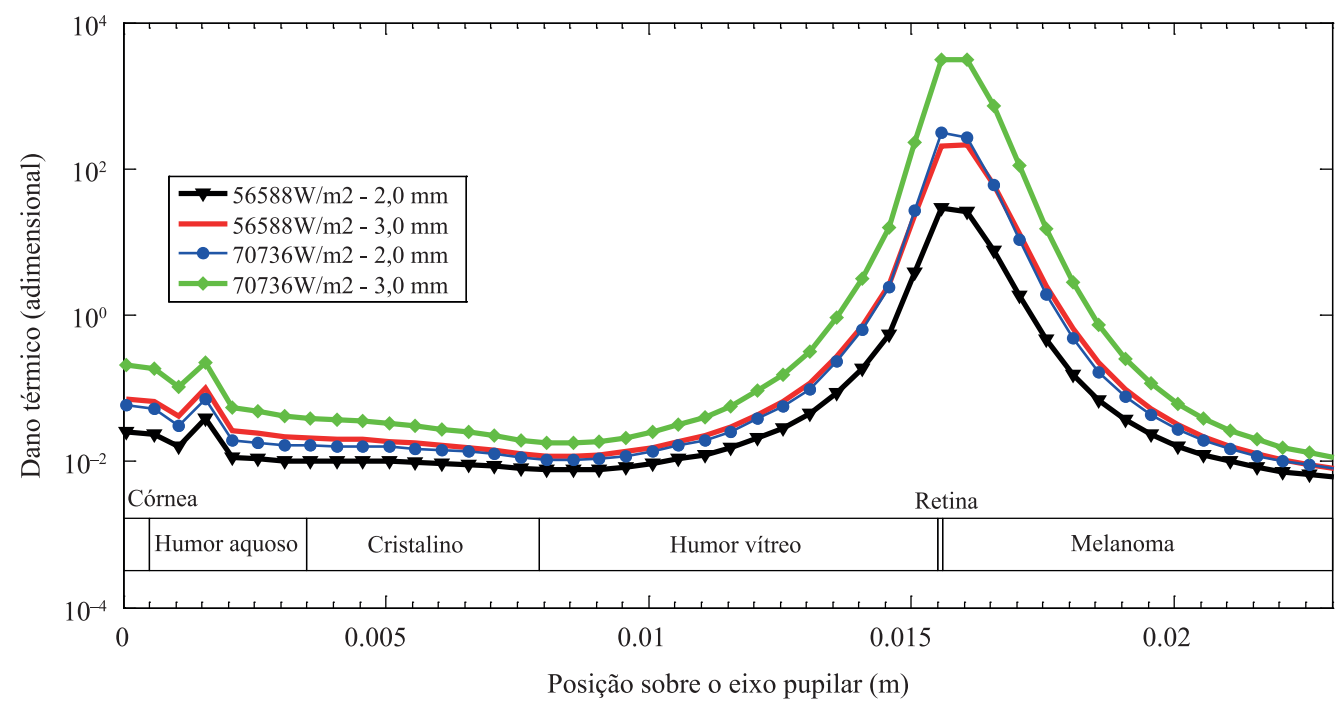

Figura 8. Dano térmico no instante $t=120$ segundos ao longo do eixo pupilar para o olho irradiado durante 60 segundos com laser de diferentes intensidades e diâmetros do feixe.

Figure 8. Thermal damage at $t=120$ seconds along the pupillary axis into the eye irradiated for 60 seconds with different intensity and laser beam diameter.

de $56.588 \mathrm{~W} / \mathrm{m}^{2}$ e $70.736 \mathrm{~W} / \mathrm{m}^{2}$ com diâmetros dos feixes iguais a 2,0 $\mathrm{mm}$ e $3,0 \mathrm{~mm}$. Ela mostra que a radiação do laser atravessou várias camadas do olho (córnea, humor aquoso, cristalino e humor vítreo) sem causar dano térmico irreversível a esses tecidos, exceto para uma pequena porção do humor vítreo que fica próxima do tumor. Isto ocorreu devido aos pequenos valores dos coeficientes de absorção destas camadas para a radiação infravermelha do laser de diodo. Ela mostra, também, que a profundidade do dano variou com a quantidade de energia entregue ao tecido, aqui representada pelo diâmetro do feixe e pela intensidade do laser.

Tecidos vivos sofrem dano térmico irreversível quando a função dano atinge o valor $\Omega=1$ (Henriques e Moritz, 1947). O dano atingiu tal valor a uma profundidade de $2,55 \mathrm{~mm}$ dentro do melanoma para a irradiação com laser de intensidade de $56.588 \mathrm{~W} / \mathrm{m}^{2}$ e diâmetro do feixe igual a 2,0 $\mathrm{mm}$. Para a mesma intensidade, mas com diâmetro do feixe igual a $3,0 \mathrm{~mm}$ o dano irreversível se estendeu até uma profundidade de $3,77 \mathrm{~mm}$. Quando foi utilizado um laser com intensidade de $70.736 \mathrm{~W} / \mathrm{m}^{2}$ e diâmetro do feixe igual a 2,0 $\mathrm{mm}$ o dano irreversível alcançou uma profundidade de $3,52 \mathrm{~mm}$ dentro do tumor; para a mesma intensidade, porém com um feixe de diâmetro igual a 3,0 mm o dano irreversível se estendeu até uma profundidade de $4,86 \mathrm{~mm}$.

\section{Discussão}

De forma geral, os resultados numércos apresentados neste artigo podem ser considerados satisfatórios.
Esta técnica foi verificada para resolver problemas de natureza semelhante em estudos prévios (Guimarães, 2003; Silva, 2004). Contudo, ainda é necessário aperfeiçoar o método quanto ao padrão geométrico utilizado, assim como em termos da aplicação da radiação laser, em uma quantidade maior de pontos. Torna-se necessário, também, a obtenção de parâmetros físicos mais precisos. Uma vez que existe grande carência de informações precisas sobre a densidade, o calor específico, a condutividade térmica, a taxa de perfusão sanguínea e o coeficiente de absorção para os vários tecidos biológicos vivos, que são necessários para que os resultados obtidos por meio das simulações possam representar as grandezas calculadas com maior margem de confiança.

Deve-se observar que, tanto no tumor quanto numa pequena porção do humor vítreo, foram encontradas temperaturas acima de $65^{\circ} \mathrm{C}$, indicando uma possível ocorrência de coagulação, e portanto, fora do intervalo considerado para a termoterapia transpupilar. No humor vítreo, estas elevadas temperaturas ocasionaram um dano inesperado ao tecido em questão. Essa ocorrência se deu, provavelmente, porque nesta simulação as regiões foram consideradas sólidas, estáticas e sem deformação e, também, devido aos valores aproximados que foram utilizados para os coeficientes de absorção, principalmente nos tecidos potencialmente espalhadores (retina, tumor e coroide), pois o coeficiente de absorção é um parâmetro crítico no modelo podendo alterar os resultados de forma significativa.

Foram realizadas análises de sensibilidade e verificou-se que o valor do coeficiente de absorção da coroide não influenciou os resultados obtidos para a 
temperatura, para o dano térmico e para a profundidade do dano térmico. Isso ocorreu provavelmente porque a intensidade da radiação que atingiu a coroide foi $0,0016 \%$ da intensidade na superfície do melanoma, não sendo suficiente para provocar alterações nessa região.

Para avaliar a influência do coeficiente de absorção no melanoma sobre os valores calculados, foram realizadas análises de sensibilidade para a profundidade do dano térmico com relação à esse parâmetro. Foi observado que a profundidade do dano varia bastante quando varia o coeficiente de absorção. Duplicando o valor do coeficiente de absorção, o valor do dano térmico na superfície do tumor foi $12,1 \%$ maior e a profundidade do dano reduziu-se para $62,5 \%$. Triplicando o valor do coeficiente de absorção, o valor do dano térmico foi $17,0 \%$ maior e a profundidade do dano reduziu-se para $46,5 \%$ do valor inicial.

Sabe-se que um dos efeitos da TTT é uma redução nas dimensões do tumor devido ao processo de desnaturação que ocorre em suas células. Pois bem, essa deformação do tumor não foi simulada; fazendo com que um determinado ponto do domínio que inicialmente pertencia ao tumor e que sofreu o processo de desnaturação, continue pertencendo ao tumor, absorvendo energia na mesma razão e, por conseguinte, atingindo temperaturas mais elevadas do que as esperadas. Então, uma maior quantidade de calor foi difundida, partindo dessa região (no tumor), para as regiões fronteiriças (no humor vítreo) acarretando temperaturas mais elevadas e causando dano térmico irreversível nessa porção do humor vítreo.

Vale ressaltar que programas de CFD resolvem problemas tridimensionais de forma satisfatória. Porém, essa análise foi realizada considerando um domínio bidimensional porque o exame de ultrassom do paciente forneceu apenas dados geométricos em duas dimensões.

\section{Conclusões}

Considerando-se as observações descritas neste artigo, podemos concluir que é possível determinar, a partir de mais estudos e desenvolvimentos, o intervalo de tempo necessário para a radiação, com uma determinada intensidade, produzir danos térmicos irreversíveis ao tecido tumoral, causando os menores prejuízos possíveis aos tecidos sadios. Os resultados apresentados sugerem que, mesmo com as incertezas relativas às propriedades termofísicas e coeficientes de absorção, este procedimento para avaliar a quantidade de energia a ser depositada no tumor em função das dimensões do mesmo poderá ser utilizado como um indicador que auxiliará na escolha da potência e diâmetro do feixe do laser nas aplicações de TTT.
Para trabalhos futuros, e para introduzir maior realismo ao modelo, sugerimos a inclusão da retina como uma camada diferente com as suas respectivas propriedades, analisando as possíveis diferenças entre esse e o novo modelo; considerar o espalhamento da radiação na retina, na coroide e no tumor, pois a aproximação utilizada nesse trabalho pode ser inadequada tendo em vista que, dependendo do tecido biológico, o coeficiente de atenuação pode diferir largamente do coeficiente de absorção, além de que o mesmo demonstrou ser um parâmetro que influenciou fortemente o valor da profundidade do dano térmico. Sugerimos, também, adotar uma estratégia computacional que possibilite a simulação do encolhimento do tumor à medida que ocorre a desnaturação das suas células.

Para o paciente analisado, existe uma simetria quase total do tumor em relação ao eixo pupilar. Por conta dessa simetria, sugerimos o desenvolvimento de uma formulação apropriada do MVF (Método dos Volumes Finitos) para lidar com modelos axisimétricos.

\section{Referências}

Amara EH. Numerical investigation on thermal effects of laser ocular media interaction. International Journal of Heat and Mass Transfer.1995; 38(13):2479-88. http://dx.doi. org/10.1016/0017-9310(94)00353-W

Arcieri ES, Fonseca D, França ET, Braga EF, Ferreira MA. Estudo de melanoma de coroide na Universidade Federal de Uberlândia. Arquivos Brasileiros de Oftalmologia. 2002; 65:89-93. http://dx.doi.org/10.1590/ S0004-27492002000100017

Charny CK. Mathematical models of bioheat transfer. In: Cho YI, editor. Advances in Heat Transfer - Bioengineering Heat Transfer. Boston: Academic Press Inc; 1992. p. 19-155. http://dx.doi.org/10.1016/S0065-2717(08)70344-7

Cheong W, Prahl SA, Welch AJ. A review of the optical properties of biological tissues. Journal of Quantum Electronics. 1990; 26(12):2166-85. http://dx.doi. org/10.1109/3.64354

Chen MM, Holmes KR. Microvascular contributions in tissue heat transfer. Annals of the New York Academy of Science. 1980; 325:137-50. http://dx.doi. org/10.1111/j.1749-6632.1980.tb50742.x

Cunha AAF, Rodrigues NHT, Almeida GA, Picanço BC, Netto JA. Melanomas de corpo ciliar e coroide: relato de caso. Arquivos Brasileiros de Oftalmologia. 2010; 73(2):193-6. PMid:20549054. http://dx.doi.org/10.1590/S000427492010000200020

Diller KP. Modeling of bioheat transfer processes at high and low temperatures. In: Cho YI, editor. Advances in Heat Transfer. Boston: Academic Press Inc; 1992. p.157-357.

Emery AF, Kramar P, Guy AW, Lin JC. Microwave induced temperature rises in rabbit eye during hyperthermic treatment. 
Journal of Heat Transfer. 1975; 97:123-28. http://dx.doi. org/10.1115/1.3450259

Forrester JV, Dick AD, McMenamin P, Lee W. The eye: Basic sciences in Practice. 2nd ed. Philadelphia: WB Saunders; 2002.

Guimarães CSC. Modelagem computacional da biotransferência de calor no tratamento por hipertermia em tumores de duodeno através do método dos volumes finitos em malhas não estruturadas [dissertação]. Recife: Universidade Federal de Pernambuco; 2003. 80 p.

Henriques FC, Moritz AR. Studies of thermal injury, I. Conduction of heat to and through the skin and the temperature attained therein. A theoretical and an experimental investigation. American Journal of Pathology. 1947; 23:531-49.

Journée-de Korver JG, Oosterhuis JA, Wolff-Rouendaal D, Kemme H. Histopathological findings in human choroidal melanomas after transpupillary thermotherapy. British Journal of Ophthalmology. 1997; 81:234-39. PMid:9135389. http:// dx.doi.org/10.1136/bjo.81.3.234

Kramar P, Harris C, Emery AF, Guy AW. Acute microwave irradiation and cataract formation in rabbits and monkeys. Journal of the Microwave Power. 1978; 13(3):239-88. PMid:108401.

Lagendijk JJW. A mathematical model to calculate temperature distributions in human and rabbit eyes during hyperthermic treatment. Physics in Medicine and Biology. 1982; 27(11):1301-11. PMid:7178232. http:// dx.doi.org/10.1088/0031-9155/27/11/001

Maliska CR. Discretização coincidente com a fronteira. In: Transferência de Calor e Mecânica dos Fluidos Computacional. Rio de Janeiro: LTC; 1995. p. 198-201.

Narasimhan A, Jha KK, Gopal L. Transient simulations of heat transfer in human eye undergoing laser surgery. International Journal of Heat and Mass Transfer. 2009; 53:482-90. http:// dx.doi.org/10.1016/j.ijheatmasstransfer.2009.09.007

Newsom RSB, McAlister JC, Saeed M, McHugh JDA. Transpupillary thermotherapy (TTT) for the treatment of choroidal neovascularisation. British Journal of Ophthalmology. 2001; 85:173-8. PMid:11159481 PMCid:1723824. http://dx.doi.org/10.1136/bjo.85.2.173

$\mathrm{Ng}$ EYK, Ooi EH. FEM simulation of the eye structure with bioheat analysis. Computer Methods and Programs in Biomedicine. 2006; 82(3):268-76. PMid:16682096. http:// dx.doi.org/10.1016/j.cmpb.2006.04.001

Pennes HH. Analysis of tissue and arterial blood temperatures in the resting forearm. Journal of Applied Physiology. 1948; 1:93-122. PMid:18887578.

Rivolta B, Inzoli F, Mantero S, Severini A. Evaluation of temperature distribution during hyperthermic treatment in biliary tumors: a computational approach. Journal of Biomechanical Engineering. 1999; 121(2):141-7. PMid:10211446. http://dx.doi.org/10.1115/1.2835095

Roizenblatt J, Rosa AAM. Termoterapia transpupilar como opção terapêutica para hemangiomas circunscrito de coroide - Relato de caso. Arquivos Brasileiros de
Oftalmologia. 2002; 65:257-60. http://dx.doi.org/10.1590/ S0004-27492002000200018

Rol P, Fankhauser F, Giger H, Dürr U, Kwasniewska S. Transpupillar laser phototherapy for retinal and choroidal tumors: a rational approach. Graefe's Archive for Clinical and Experimental Ophthalmology. 2000; 238:249-72. http:// dx.doi.org/10.1007/s004170050352

Santos SKSL. Desenvolvimento de uma ferramenta para aquisição de pontos em imagens digitais para a utilização no cálculo de temperaturas através de modelagem computacional [dissertação]. Recife: Universidade Federal de Pernambuco; 2007. 75 p.

Scott JA. A finite element model of heat transport in the human eye. Physics in Medicine and Biology. 1988; 33(2):227-41. PMid:3362966. http://dx.doi.org/10.1088/0031-9155/33/2/003

Shields CL, Shields JA, Cater J, Lois N, Edelstein C, Gündüz K, Mercado G. Transpupillary thermotherapy for choroidal melanoma: tumor control and visual results of 100 consecutive cases. Ophthalmology. 1998; 105:581-90. http:// dx.doi.org/10.1016/S0161-6420(98)94008-8

Shitzer A, Eberh RC. Heat transfer in medicine and biology: analysis and applications. New York: Plenum Press; 1985.

Silva GMLL. Análise da biotransferência de calor nos tecidos oculares devido à presença de implantes retinianos através da utilização do método dos volumes finitos em malhas não-estruturadas [dissertação]. Recife: Universidade Federal de Pernambuco; 2004. 72 p.

Smerson D. Anatomy of the eye and orbit. Current Anaesthesia \& Critical Care. 2000; 2: 286-92. http://dx.doi.org/10.1054/ cacc. 2000.0296

Stoffelns BM. Primary transpupillary thermotherapy (TTT) for malignant choroidal melanoma. Acta Ophthalmologica Scandinavica. 2002; 80:25-31. PMid:11906300. http:// dx.doi.org/10.1034/j.1600-0420.2002.800106.x

Valenzuela HG. Melanoma choroidal: Treatment \& medication. Medscape's Continually Updated Clinical Reference [Internet]. 2009 [cited 2010 Feb 05]. Available from: http://emedicine.medscape.com

Van den Berg TJTP, Spekreijse H. Near infrared light absorption in the human eye media. Vision Research. 1997; 37(2):249-53. http://dx.doi.org/10.1016/ S0042-6989(96)00120-4

Weinbaum S, Jiji LM, Lemons DE. Theory and experiment for the effect of vascular microstructure on surface tissue heat transfer - part I: anatomical foundation and model conceptualization. Journal of Biomechanical Engineering. 1984; 106:321-30. PMid:6513527. http:// dx.doi.org/10.1115/1.3138501

Welch AJ. Laser irradiation of tissue. In: Shitzer A, Eberhart RC, editors. Heat Transfer in Medicine and Biology - Analysis and Applications. Nova York: Plenum Press; 1985. p. 135-84.

Wulff W. The energy conservation equation for living tissue. IEEE Transactions on Biomedical Engineering. 1974; 21:494-5. http://dx.doi.org/10.1109/ TBME.1974.324342 


\section{Autores}

\section{José Duarte da Silva*}

Instituto Federal de Educação, Ciência e Tecnologia de Pernambuco - IFPE, Av. Professor Luiz Freire, 500,

Cidade Universitária, CEP 50740-540, Recife, PE, Brasil.

Paulo Roberto Maciel Lyra, Rita de Cássia Fernandes de Lima

Departamento de Engenharia Mecânica, Universidade Federal de Pernambuco - UFPE, Av. Acadêmico Hélio Ramos, s/n, Cidade Universitária, CEP 50740-530, Recife, PE, Brasil. 